\title{
Desigualdades sociodemográficas nos fatores de risco e proteção para doenças crônicas não transmissíveis: inquérito telefônico em Campinas, São Paulo
}

doi: 10.5123/S1679-49742015000100002

\author{
Sociodemographic inequalities in non communicable chronic disease risk and protection \\ factors: telephone survey in Campinas, São Paulo, Brazil
}

\author{
Priscila Maria Stolses Bergamo Francisco \\ Universidade Estadual de Campinas, Faculdade de Ciências Médicas, Departamento de Saúde Coletiva, Campinas-SP, Brasil \\ Neuber José Segri \\ Universidade Federal de Mato Grosso, Departamento de Estatística, Cuiabá-MT, Brasil \\ Marilisa Berti de Azevedo Barros \\ Universidade Estadual de Campinas, Faculdade de Ciências Médicas, Departamento de Saúde Coletiva, Campinas-SP, Brasil \\ Deborah Carvalho Malta \\ Ministério da Saúde, Secretaria de Vigilância em Saúde, Brasília-DF, Brasil \\ Universidade Federal de Minas Gerais, Departamento de Enfermagem Materno-Infantil e Saúde Pública, Belo Horizonte-MG, Brasil
}

\section{Resumo}

Objetivo: identificar e analisar desigualdades sociodemográficas em fatores de risco e proteção para a saúde. Métodos: estudo transversal de base populacional realizado em Campinas-SP, com 2.015 adultos ( $\geq 18$ anos) entrevistados pelo Sistema de Vigilância de Fatores de Risco e Proteção para Doenças Crônicas por Inquérito Telefônico - Vigitel - em 2008; estimaram-se prevalências e razões de prevalência de fatores de risco e proteção e respectivos intervalos de confiança de 95\%, sobre amostra probabilística coberta por telefonia fixa. Resultados: observaram-se desigualdades sociodemográficas nos fatores de risco e proteção avaliados: fumantes e ex-fumantes corresponderam a 17,1\% e 20,7\%, respectivamente, aproximados 47,0\% apresentaram excesso de peso e apenas 13,4\% referiram prática de atividade física; em geral, os fatores de risco foram mais prevalentes entre homens e indivíduos menos escolarizados. Conclusão: os distintos comportamentos em saúde observados contribuem para a definição de prioridades, alocação de recursos e delineamento de programas de redução de desigualdades sociodemográficas na Saúde.

Palavras-chave: Desigualdades em Saúde; Inquéritos de Saúde; Vigilância Epidemiológica; Fatores de Risco; Doença Crônica.

\begin{abstract}
Objective: to identify and analyze sociodemographic disparities in health risk and protection. Methods: this was a population-based cross-sectional study conducted in Campinas-SP with 2,015 adults (aged $\geq 18$ years) interviewed via Telephone-based Surveillance of Chronic Disease Risk and Protection Factors in 2008; risk and protection factor prevalence and prevalence ratios and respective 95\% confidence intervals were estimated from a probabilistic sample of people with residential telephone lines. Results: inequalities were found in the assessed risk and protection factors; smokers and former smokers accounted for $17.1 \%$ and $20.7 \%$ of the interviewees respectively, some $47.0 \%$ were overweight, and only $13.4 \%$ reported physical activity; in general risk factors were more prevalent among men and individuals with less education. Conclusion: the different bealth behaviors observed contribute to priority setting, resource allocation and program design aimed at reducing sociodemographic bealth inequalities in this population.
\end{abstract}

Key words: Health Inequalities; Health Surveys; Epidemiological Surveillance; Risk Factors; Chronic Disease.

Endereço para correspondência:

Priscila Maria Stolses Bergamo Francisco - Universidade Estadual de Campinas, Faculdade de Ciências Médicas, Departamento de Saúde Coletiva, Rua Tessália Vieira de Camargo, nº 126, Cidade Universitária, Campinas-SP, Brasil. CEP: $13083-887$

E-mail:primaria@fcm.unicamp.br 


\section{Introdução}

As doenças crônicas não transmissíveis (DCNT) representam um dos maiores problemas de Saúde Pública global na atualidade. A proporção de óbitos por DCNT é muito mais elevada nos países de baixa e média renda. ${ }^{1}$ Dados relativos a 2007, corrigidos para causas mal definidas e sub-registro, apontaram que, no Brasil, cerca de $72 \%$ de todas as mortes foram atribuídas a DCNT: doenças cardiovasculares, respiratórias crônicas, diabetes, câncer e outras, incluindo doença renal. ${ }^{2}$

Essas doenças e seus fatores de risco afetam pessoas de todos os grupos socioeconômicos, especialmente aquelas mais vulneráveis, como os idosos e indivíduos de famílias com baixo nível educacional ou menor renda. ${ }^{3}$ No Brasil, inquéritos populacionais de saúde têm demonstrado maior frequência de tabagismo em indivíduos de ambos os sexos, e de obesidade entre mulheres e pessoas com menor nível educacional, ${ }^{4}$ além de dietas pouco saudáveis. ${ }^{5}$

\section{As desigualdades influenciam na qualidade de vida e na capacidade de ser e agir de grupos sociais específicos.}

Os fatores de risco modificáveis constituem a causa expressa de grande parte da carga das doenças, tanto na mortalidade geral quanto nas mortes prematuras e incapacidades decorrentes ${ }^{6}$ relacionadas às DCNT. Parte dessa carga resulta das condições em que as pessoas nascem, vivem, trabalham e envelhecem, ou seja, da atuação dos determinantes sociais sobre a saúde da população. Determinantes sociais estabelecem posições socioeconômicas, as quais, por sua vez, geram hierarquias de poder e de acesso a recursos básicos, ${ }^{7}$ entre eles os relacionados à promoção da saúde e prevenção de agravos.

Estudos apontam que parte da redução observada na incidência e mortalidade por DCNT se deve a um maior controle dos fatores de risco modificáveis. ${ }^{6,8,9}$ Atualmente, muito se sabe sobre as causas evitáveis de doenças não transmissíveis e as ações necessárias para sua prevenção e controle estão bem documentadas, o suficiente para que sejam avaliadas e implementadas. ${ }^{6,7,9}$
Segundo Olsen e colaboradores, ${ }^{10}$ parcela significativa das pesquisas sobre o tema procedem de países desenvolvidos, e a implementação de ações para o controle desses fatores de risco requer 0 conhecimento e a consideração de realidades locais. As DCNT resultam de múltiplas causas, atuantes em conjunto, para provocar efeitos que podem ser diferentes segundo as populações abordadas, enquanto inseridas em contextos distintos, ${ }^{10}$ onde as desigualdades influenciam na qualidade de vida e na capacidade de ser e agir de grupos sociais específicos. São especificidades relevantes, a serem consideradas na elaboração de estratégias de prevenção e controle dessas doenças.

Os inquéritos populacionais de saúde viabilizam a obtenção de dados sobre várias dimensões relativas ao processo saúde-doença e produzem informações essenciais para a orientação e formulação de ações, estratégias e políticas de saúde, assim como para a avaliação do impacto de políticas sociais e intervenções na área da Saúde. ${ }^{11}$

No ano de 2006, o Ministério da Saúde do Brasil implantou um sistema de inquérito por telefone sobre fatores de risco para DCNT, expandindo esse trabalho de pesquisa para todas as capitais brasileiras e o Distrito Federal. ${ }^{12}$ Entrevistas realizadas por telefone apresentam vantagens, frente aos diversos métodos de coleta de dados empregados em inquéritos, pela presteza, menor custo e elevada taxa de resposta. ${ }^{4,13}$

No ano de 2008, o Ministério da Saúde selecionou o município de Campinas-SP para a realização do inquérito telefônico (Sistema de Vigilância de Fatores de Risco e Proteção para Doenças Crônicas por Inquérito Telefônico - Vigitel) porque, no mesmo ano, seria realizado um inquérito domiciliar de saúde na cidade (Inquérito de Saúde no Município de Campinas - ISACamp), possibilitando comparações de alguns indicadores, obtidos a partir de questões semelhantes aplicadas em ambas as modalidades de inquérito. ${ }^{13}$ Cabe ressaltar que os dados sobre os fatores de risco e proteção coletados por essa pesquisa ainda não foram divulgados em sua totalidade.

0 objetivo deste estudo foi identificar e analisar desigualdades em fatores de risco e proteção para a saúde segundo sexo, idade e escolaridade na população adulta ( $\geq 18$ anos) de Campinas, estado de São Paulo, Brasil. 


\section{Métodos}

Para a realização do presente estudo, foram incluídos 2.015 indivíduos com 18 ou mais anos de idade, entrevistados pelo Vigitel no ano de 2008, em Campinas-SP.

O município de Campinas-SP localiza-se a noroeste e a cerca de $100 \mathrm{~km}$ da capital do estado, possui uma área de $795 \mathrm{~km}^{2}$ e constitui um polo industrial e tecnológico. De acordo com a Fundação Instituto Brasileiro de Geografia e Estatística (IBGE), em 2008, o produto interno bruto (PIB) per capita era de $\mathrm{R} \$ 27.731,98 \mathrm{e}$ 0 índice de desenvolvimento humano (IDH) de renda era de 0,845 , no município. ${ }^{14}$ Segundo dados do último censo demográfico, realizado em 2010 , a população de Campinas-SP era de 1.080.113 habitantes, dos quais cerca de 12,0\% constituídos por pessoas com 60 ou mais anos de idade. ${ }^{14}$

0 procedimento de amostragem visou a obtenção de amostra probabilística da população adulta $(\geq 18$ anos) residente em domicílios servidos por, pelo menos, uma linha telefônica fixa no ano de $2008 .{ }^{12}$ A seleção da amostra foi realizada pelo sorteio das linhas telefônicas, a partir de cadastro eletrônico com 228.091 linhas residenciais das empresas telefônicas que cobriam o município, e dos moradores adultos. Em Campinas-SP, foram realizadas ligações para 4.800 linhas telefônicas distribuídas em 24 réplicas, identificando-se 2.773 linhas elegíveis. ${ }^{13}$ Para cada linha elegível, obtido o consentimento dos seus usuários em participar da pesquisa, procedeu-se à enumeração dos indivíduos que residiam no domicílio selecionado e ao sorteio, por meio de tabela de números aleatórios, de um desses moradores, para então proceder à entrevista. ${ }^{12}$

As entrevistas foram realizadas de abril a dezembro de 2008 e 0 instrumento de coleta contemplou questões sobre características sociodemográficas dos indivíduos, informações sobre hábito alimentar, atividade física, peso e altura recordados, além da frequência de consumo de cigarros e de bebidas alcoólicas, da autoavaliação do estado de saúde e da referência a diagnóstico médico anterior de algumas doenças. ${ }^{12}$

Os dados analisados incorporaram o ajuste de pós-estratificação que considerou a estrutura etária da população do censo demográfico de 2000 , calculado como a razão entre a frequência relativa de indivíduos da população e na amostra, considerando-se 36 estra- tos segundo sexo (masculino e feminino), faixas etárias (18-24, 25-34, 35-44, 45-54, 55-64 e 65 ou mais anos) e escolaridade ( 0 a 8,9 a 11 e 12 ou mais anos de estudo). Além disso, incorporaram-se outros dois componentes - inverso do número de linhas telefônicas no domicílio do entrevistado e número de adultos no domicílio do entrevistado - para compensar o viés decorrente da não cobertura universal de telefonia fixa no município, compondo o peso final aplicado a cada indivíduo entrevistado. ${ }^{12}$ Neste estudo, entre os fatores de risco, foram avaliados:

- Hábito de fumar - percentual de indivíduos que fumavam no momento da entrevista, independentemente da frequência e intensidade do hábito.

- Consumo abusivo de bebidas alcoólicas - 5 ou mais doses para homens e 4 ou mais doses para mulheres em uma mesma ocasião, nos últimos 30 dias.

- Estado nutricional ${ }^{15}$ - excesso de peso, caracterizado pelo percentual de indivíduos com índice de massa corporal (IMC) igual ou superior a $25 \mathrm{~kg} /$ $\mathrm{m}^{2}$; e obesidade, considerando-se o IMC igual ou superior a $30 \mathrm{~kg} / \mathrm{m}^{2}$.

- Hábito alimentar - consumo de refrigerante ou suco artificial com açúcar por 5 ou mais dias na semana e de carnes com excesso de gordura (percentual de indivíduos que costumam consumir carne vermelha com gordura aparente ou frango sem a remoção da pele).

- Referência a diagnóstico médico de hipertensão arterial, diabetes e dislipidemia.

$\mathrm{Na}$ avaliação dos fatores de proteção, foram considerados:

- Cessação do tabagismo, considerando-se o percentual de ex-fumantes.

- Consumo regular de frutas, legumes e verduras (FLV) - consumo referido de FLV em 5 ou mais dias da semana e consumo de FLV conforme recomendado ( 5 ou mais porções diárias de FLV em 5 ou mais dias da semana).

- Atividade física suficiente no período de lazer prática de atividade física de intensidade leve ou moderada por, pelo menos, 30 minutos diários em 5 ou mais dias da semana, ou de atividades de intensidade vigorosa por, pelo menos, 20 minutos diários em 3 ou mais dias da semana.

Para a população adulta feminina, também foi avaliada a realização de exames preventivos: citologia oncótica nos últimos 3 anos para as mulheres entre 25 
e 59 anos de idade, e mamografia nos últimos 2 anos para aquelas entre 50 e 69 anos de idade.

Foram estimadas as prevalências e respectivos intervalos de confiança de $95 \%$ para os fatores de risco e proteção, segundo sexo, faixas etárias (18 a 39,40 a 59 e 60 ou mais anos) e escolaridade ( 0 a 8 , 9 a 11 e 12 ou mais anos de estudo). A comparação entre as proporções foi realizada pelos testes do qui-quadrado de Pearson (Rao-Scott) e de tendência linear, considerando-se estatisticamente significativos aqueles com $\mathrm{p}<0,05$; e pelas razões de prevalência ajustadas, calculadas pela regressão de Poisson. As análises foram realizadas pelo aplicativo Stata $11.0 \mathrm{e}$ consideraram todos os fatores de ponderação.

O consentimento livre e esclarecido foi substituído pela aquiescência verbal, obtida por ocasião dos contatos telefônicos com os entrevistados. 0 projeto de implantação do Vigitel foi aprovado pelo Comissão Nacional de Ética em Pesquisa, do Ministério da Saúde. ${ }^{12}$

\section{Resultados}

A média de idade dos adultos residentes em Campinas-SP entrevistados pelo Vigitel $(\mathrm{n}=2.015)$ foi de 39,9 anos (IC ${ }_{95 \%}$ : 38,9-40,9); mais da metade (52,1\%) eram mulheres, com predomínio de adultos jovens e maior proporção $(54,5 \%)$ de pessoas com até 8 anos de estudo (Tabela 1). A proporção de resposta obtida pelo inquérito foi de $72,7 \%$ (entrevistas realizadas/linhas elegíveis).
As prevalências dos fatores de risco e proteção para DCNT na população adulta geral, segundo sexo, são apresentadas na Tabela 2. Os percentuais de fumantes e ex-fumantes observados foram de $17,1 \%$ e $20,7 \%$ respectivamente, sendo mais elevados entre os homens. O consumo abusivo de bebidas alcoólicas foi verificado em 15,0\% da população, sendo significativamente menor entre as mulheres $\left(\mathrm{RP}=0,33 ; \mathrm{IC}_{95 \%}: 0,24-0,45\right)$. A prevalência de excesso de peso foi de $47,3 \%$, e de obesidade, de cerca de $14,0 \%$, sendo que o excesso de peso foi maior entre os homens. Quanto à alimentação, entre todos os adultos, observou-se prevalência de 34,4\% para o consumo regular de frutas, verduras e legumes - FVL -, e de $22,1 \%$ para o consumo recomendado de FVL, ou seja, 5 ou mais porções diárias em 5 ou mais dias da semana. Hábitos alimentares mais saudáveis foram observados entre as mulheres. A frequência de adultos que praticavam atividade física no tempo livre foi mais elevada nos homens. Em relação à hipertensão arterial, diabetes e dislipidemia referidas pelos entrevistados a partir de diagnósticos médicos prévios, as prevalências observadas foram de 22,3\%, 6,0\% e 16,6\% respectivamente. Nas mulheres, a prevalência de dislipidemia foi maior do que nos homens ( $\left.\mathrm{RP}=1,29 ; \mathrm{IC}_{95 \%}: 1,02-1,62\right)$; para as demais doenças crônicas, não foi observada diferença entre os sexos. 0 percentual de mulheres que referiram realização do exame de citologia oncótica foi de $95,4 \%$, e a frequência de mulheres que realizaram mamografia nos últimos 2 anos foi de $91,0 \%$.

\section{Tabela 1 - Distribuição percentual da população adulta ( $\geq 18$ anos) $(n=2.015)$ segundo características sociodemográficas no município de Campinas, São Paulo, 2008}

\begin{tabular}{lrr}
\hline Variáveis e categorias & $\mathbf{n}^{\mathrm{a}}$ & $(\%)^{\mathrm{b}}$ \\
\hline Sexo & & \\
\hline Masculino & 851 & $47,9(44,8-51,0)$ \\
$\quad$ Feminino & 1.164 & $52,1(49,0-55,2)$ \\
\hline Faixa etária (em anos) & & \\
\hline $18-39$ & 846 & $53,8(50,8-56,7)$ \\
$40-59$ & 756 & $32,6(30,0-35,2)$ \\
$\geq 60$ & 413 & $13,6(12,1-15,2)$ \\
\hline Escolaridade (em anos de estudo) & & \\
\hline $0-8$ & 711 & $54,5(51,5-57,4)$ \\
$9-11$ & 712 & $25,7(23,5-27,9)$ \\
$\geq 12$ & 592 & $19,8(17,9-21,7)$ \\
\hline
\end{tabular}

a) n:número de indivíduos na amostra não ponderada

b) Percentual ponderado para ajustar a distribuição sociodemográfica da amostra do Sistema de Vigilância de Fatores de Risco e Proteção para Doenças (rônicas por Inquérito Telefônico (Vigitel) à distribuição da população adulta na cidade Campinas-SP segundo o censo demográfico de 2000 
Tabela 2 - Prevalência e razão de prevalência de fatores de risco e proteção para doenças crônicas não transmissíveis em adultos ( $\geq 18$ anos) segundo sexo no município de Campinas, São Paulo, 2008

\begin{tabular}{|c|c|c|c|c|c|c|c|c|}
\hline \multirow{3}{*}{ Indicadores } & \multirow{2}{*}{\multicolumn{2}{|c|}{ Total }} & \multicolumn{4}{|c|}{ Sexo } & \multicolumn{2}{|c|}{$\mathrm{RP}_{\text {Ajustada }}{ }^{\mathrm{a}}(2) /(1)$} \\
\hline & & & \multicolumn{2}{|c|}{ Masculino (1) } & \multicolumn{2}{|c|}{ Feminino (2) } & \multirow[b]{2}{*}{$\mathbf{R P}$} & \multirow[b]{2}{*}{$I_{95 \%}{ }^{b}$} \\
\hline & $\%$ & $I_{95 \%}{ }^{b}$ & $\%$ & $I_{95 \%}{ }^{b}$ & $\%$ & $I_{95 \%}{ }^{b}$ & & \\
\hline \multicolumn{9}{|l|}{ Hábito de fumar } \\
\hline Fumantesc & 17,1 & $14,5-19,6$ & 21,1 & $16,9-25,4$ & 13,3 & $10,3-16,2$ & 0,63 & $0,47-0,85$ \\
\hline Ex-fumantes ${ }^{c}$ & 20,7 & $18,5-22,9$ & 24,2 & $20,7-27,7$ & 17,4 & $14,6-20,2$ & 0,70 & $0,56-0,86$ \\
\hline Consumo abusivo de álcoolc & 15,0 & $12,8-17,3$ & 23,2 & $19,1-27,2$ & 7,6 & $5,6-9,5$ & 0,33 & $0,24-0,45$ \\
\hline \multicolumn{9}{|l|}{ Estado nutricional } \\
\hline Excesso de pesoc & 47,3 & $44,2-50,5$ & 52,3 & $47,5-57,1$ & 42,3 & $38,3-46,3$ & 0,80 & $0,70-0,91$ \\
\hline Obesidade & 14,1 & $12,1-16,2$ & 12,8 & $9,9-15,8$ & 15,5 & $12,5-18,4$ & 1,18 & $0,88-1,59$ \\
\hline \multicolumn{9}{|l|}{ Hábito alimentar } \\
\hline FVL ${ }^{d}$ regularc & 34,4 & $31,5-37,3$ & 29,4 & $24,9-33,8$ & 39,0 & $35,2-42,8$ & 1,31 & $1,10-1,57$ \\
\hline $\mathrm{FVL}^{\mathrm{d}}$ recomendadoc & 22,1 & $19,7-24,6$ & 18,8 & $15,3-22,3$ & 25,2 & $21,9-28,5$ & 1,32 & $1,06-1,66$ \\
\hline Refrigerantes $^{c}$ & 29,1 & $26,1-32,0$ & 33,4 & $28,7-38,2$ & 25,1 & $21,5-28,7$ & 0,77 & $0,63-0,94$ \\
\hline Carnes com excesso de gordurac & 27,9 & $25,1-30,7$ & 37,4 & $32,7-42,1$ & 19,1 & $16,1-22,2$ & 0,52 & $0,42-0,63$ \\
\hline \multicolumn{9}{|l|}{ Atividade física } \\
\hline Ativo suficiente no lazerc & 13,4 & $11,4-15,5$ & 15,7 & $12,1-19,2$ & 11,4 & $9,3-13,5$ & 0,73 & $0,55-0,97$ \\
\hline \multicolumn{9}{|l|}{ Diagnóstico médico de doenças } \\
\hline Hipertensão arterial & 22,3 & $20,0-24,6$ & 20,4 & $17,0-23,8$ & 24,1 & $21,0-27,2$ & 1,11 & $0,91-1,35$ \\
\hline Diabetes & 6,0 & $4,8-7,2$ & 4,9 & $3,3-6,5$ & 6,9 & $5,2-8,7$ & 1,30 & $0,86-1,96$ \\
\hline Dislipidemia $^{c}$ & 16,6 & $14,6-18,6$ & 14,0 & $11,3-16,6$ & 19,0 & $16,0-21,9$ & 1,29 & $1,02-1,62$ \\
\hline \multicolumn{9}{|l|}{ Exames preventivos } \\
\hline Papanicolaou ${ }^{e}$ & - & - & - & - & 95,4 & $92,9-98,0$ & - & - \\
\hline Mamografia $^{f}$ & - & - & - & - & 91,0 & $87,2-94,8$ & - & - \\
\hline
\end{tabular}

a) $\mathrm{RP}_{\text {Austadada }}$ :razão de prevalência ajustada por idade; categoria de referência - população masculina.

b) IC Ajustata intervalo de confiança de $95 \%$

c) Diferença significativa $(p<0,05)$ pelo teste do qui-quadrado

d) FVL: frutas, verduras e legumes.

e) Exame realizado em mulheres de 25 a 59 anos de idade, nos últimos 3 anos.

f) Exame realizado em mulheres de 50 a 69 anos de idade, nos últimos 2 anos.

Nota: percentual ponderado para ajustar a distribuição sociodemográfica da amostra do Vigitel à distribuição da população adulta da cidade Campinas-SP segundo o censo demográfico de 2000

Fonte:Sistema de Vigilância de Fatores de Risco e Proteção para Doenças Crônicas por Inquérito Telefônico (Vigitel)/Ministério da Saúde

Na avaliação segundo faixas etárias (Tabela 3), observou-se que a frequência de fumantes foi mais elevada nas pessoas com idade entre $40 \mathrm{e} 59$ anos, em relação àquelas mais jovens. 0 percentual de ex-fumantes aumentou significativamente com a idade. Para o excesso de peso e obesidade, maiores prevalências foram observadas entre adultos de 40 a 59 anos e idosos. Maiores frequências de consumo regular e recomendado de frutas, legumes e verduras foram verificadas nos idosos, em relação aos adultos jovens $\left(\mathrm{RP}=1,49 ; \mathrm{IC}_{95 \%}: 1,16-1,92\right)$. Por sua vez, o consumo de refrigerante ou suco artificial foi mais frequente entre os mais jovens, em relação às demais faixas etárias. Nos idosos, o consumo de carne com excesso de gordura foi menos frequente quando comparado ao de outros grupos etários, assim como o uso excessivo de álcool. A referência de diagnóstico de hipertensão e de diabetes foi mais elevada nos adultos com idade entre 40 e 59 anos $\left(\mathrm{RP}=2,80 ; \mathrm{IC}_{95 \%}\right.$ : $2,05-3,82)$ e nos idosos ( $\left.\mathrm{RP}=5,31 ; \mathrm{IC}_{95 \%}: 3,93-7,18\right)$, em relação aos mais jovens. Quanto à dislipidemia, as prevalências observadas para faixas etárias mais elevadas foram estatisticamente semelhantes.

Na Tabela 4, verifica-se maior prevalência de tabagismo nos adultos com menor escolaridade. Em 
Tabela 3 - Prevalência e razão de prevalência de fatores de risco e proteção para doenças crônicas não transmissíveis em adultos ( $\geq \mathbf{1 8}$ anos) segundo faixas etárias no município de Campinas, São Paulo, 2008

\begin{tabular}{|c|c|c|c|c|c|c|c|}
\hline \multirow{2}{*}{ Indicadores } & \multicolumn{3}{|c|}{$\begin{array}{l}\text { Faixas etárias } \\
\text { (em anos) }\end{array}$} & \multicolumn{4}{|c|}{$\mathbf{R P}_{\text {Ajustada }}{ }^{\text {a }}$} \\
\hline & $\begin{array}{l}18-39 \\
(1)\end{array}$ & $\begin{array}{l}40-59 \\
(2)\end{array}$ & $\begin{array}{l}\geq 60 \\
(3)\end{array}$ & $\begin{array}{c}\mathrm{RP} \\
(2) /(1)\end{array}$ & $\mathrm{IC}_{95 \%}^{\mathrm{b}}$ & $\begin{array}{l}\mathrm{RP} \\
(3) /(1)\end{array}$ & $\mathrm{IC}_{95 \%}^{\mathrm{b}}$ \\
\hline \multicolumn{8}{|l|}{ Hábito de fumar } \\
\hline Fumantes ${ }^{c}$ & 15,8 & 21,5 & 11,3 & 1,37 & $1,01-1,86$ & 0,74 & $0,48-1,12$ \\
\hline Ex-fumantesc & 12,7 & 28,6 & 33,2 & 2,26 & $1,74-2,93$ & 2,68 & $2,04-3,53$ \\
\hline Consumo abusivo de álcook & 16,4 & 16,1 & 7,3 & 1,00 & $0,75-1,33$ & 0,48 & $0,30-0,77$ \\
\hline \multicolumn{8}{|l|}{ Estado nutricional } \\
\hline Excesso de pesoc & 39,0 & 57,5 & 55,1 & 1,48 & $1,28-1,71$ & 1,43 & $1,21-1,69$ \\
\hline Obesidade ${ }^{c}$ & 11,2 & 16,9 & 19,0 & 1,50 & $1,07-2,10$ & 1,68 & $1,15-2,44$ \\
\hline \multicolumn{8}{|l|}{ Hábito alimentar } \\
\hline FVL' regularc & 30,6 & 36,5 & 44,2 & 1,19 & $0,98-1,43$ & 1,41 & $1,16-1,71$ \\
\hline $\mathrm{FVL}^{\mathrm{d}}$ recomendadoc & 19,5 & 23,3 & 29,7 & 1,19 & $0,94-1,52$ & 1,49 & $1,16-1,92$ \\
\hline Refrigerantes $^{c}$ & 38,0 & 22,0 & 11,2 & 0,58 & $0,47-0,72$ & 0,30 & $0,21-0,42$ \\
\hline Carnes com excesso de gordurac & 28,8 & 31,0 & 17,0 & 1,08 & $0,89-1,32$ & 0,62 & $0,46-0,83$ \\
\hline \multicolumn{8}{|l|}{ Atividade física } \\
\hline Ativo suficiente no lazer & 14,0 & 13,5 & 11,1 & 0,97 & $0,72-1,32$ & 0,81 & $0,56-1,17$ \\
\hline \multicolumn{8}{|l|}{ Diagnóstico médico de doenças } \\
\hline Hipertensão arterialc & 10,2 & 28,7 & 54,7 & 2,80 & $2,05-3,82$ & 5,31 & $3,93-7,18$ \\
\hline Diabetes $^{c}$ & 2,3 & 6,7 & 18,2 & 2,81 & $1,44-5,48$ & 7,47 & $3,93-14,21$ \\
\hline Dislipidemiac $^{c}$ & 6,2 & 26,7 & 33,5 & 4,31 & $2,92-6,36$ & 5,31 & $3,57-7,91$ \\
\hline
\end{tabular}

relação ao hábito alimentar, tanto o consumo regular quanto o recomendado de FVL foram mais elevados entre as pessoas com melhor nível de escolaridade, que também referiram menores frequências de consumo de carnes com excesso de gordura e refrigerante ou suco artificial. Entre os mais escolarizados, verificou-se uma prevalência de atividade física no lazer 57,0\% maior em relação ao grupo com menor escolaridade, independentemente do sexo e da idade $(\mathrm{RP}=1,57$; $\left.\mathrm{IC}_{95 \%}: 1,08-2,27\right)$. No que se refere às doenças investigadas, observou-se maior prevalência de hipertensão arterial nos que referiram escolaridade igual ou inferior a 8 anos de estudo. Nas mulheres, não houve diferença estatística na realização dos exames preventivos quando relacionada aos níveis de escolaridade.

\section{Discussão}

No presente estudo, foram observadas diferenças na distribuição dos fatores de risco e proteção avaliados, segundo as características sociodemográficas consideradas. De modo geral, os fatores de risco foram mais prevalentes entre homens e nos indivíduos com menor escolaridade, enquanto maiores prevalências de fatores de proteção foram verificadas nas mulheres, nos idosos e nos mais escolarizados, conforme tem sido apontado para adultos brasileiros. ${ }^{4,5,16}$ As características sociodemográficas da população estudada foram apresentadas sob uma perspectiva comparativa àquelas obtidas pelo ISACamp e constam em outra publicação. ${ }^{13}$ 
Tabela 4 - Prevalência e razão de prevalência de fatores de risco e proteção para doenças crônicas não transmissíveis em adultos ( $\geq 18$ anos) segundo escolaridade no município de Campinas, São Paulo, 2008

\begin{tabular}{|c|c|c|c|c|c|c|c|}
\hline \multirow[b]{2}{*}{ Indicadores } & \multicolumn{3}{|c|}{ Anos de estudo } & \multicolumn{4}{|c|}{$\mathbf{R P}_{\text {Ajustada }}{ }^{\mathbf{a}}$} \\
\hline & $\begin{array}{l}0-8 \\
(1)\end{array}$ & $\begin{array}{l}9-11 \\
(2)\end{array}$ & $\begin{array}{l}\geq 12 \\
(3)\end{array}$ & $\begin{array}{c}\mathrm{RP} \\
(2) /(1)\end{array}$ & IC $_{95 \%}{ }^{\mathrm{b}}$ & $\begin{array}{c}\text { RP } \\
(3) /(1)\end{array}$ & $I_{95 \%}{ }^{b}$ \\
\hline \multicolumn{8}{|l|}{ Hábito de fumar } \\
\hline Fumantes ${ }^{c}$ & 21,3 & 12,6 & 11,1 & 0,58 & $0,41-0,81$ & 0,49 & $0,35-0,68$ \\
\hline Ex-fumantes ${ }^{c}$ & 22,6 & 18,6 & 18,1 & 1,08 & $0,84-1,39$ & 0,90 & $0,70-1,16$ \\
\hline Consumo abusivo de álcoolc & 13,2 & 17,4 & 16,9 & 1,23 & $0,86-1,76$ & 1,19 & $0,85-1,67$ \\
\hline \multicolumn{8}{|l|}{ Estado nutricional } \\
\hline Excesso de peso ${ }^{c}$ & 50,0 & 43,5 & 45,3 & 0,98 & $0,83-1,15$ & 0,95 & $0,81-1,10$ \\
\hline Obesidade $^{c}$ & 16,3 & 11,4 & 12,2 & 0,79 & $0,54-1,13$ & 0,79 & $0,56-1,12$ \\
\hline \multicolumn{8}{|l|}{ Hábito alimentar } \\
\hline FVL $^{d}$ regularc & 31,1 & 34,4 & 43,6 & 1,22 & $0,98-1,53$ & 1,51 & $1,23-1,85$ \\
\hline $\mathrm{FVL}^{\mathrm{d}}$ recomendadoc & 19,3 & 21,9 & 30,4 & 1,28 & $0,95-1,72$ & 1,72 & $1,32-2,25$ \\
\hline Refrigerantes $^{c}$ & 27,7 & 36,9 & 22,8 & 1,09 & $0,88-1,36$ & 0,73 & $0,57-0,94$ \\
\hline $\begin{array}{l}\text { Carnes com excesso } \\
\text { de gordurac }\end{array}$ & 31,3 & 28,2 & 18,3 & 0,85 & $0,69-1,06$ & 0,55 & $0,43-0,70$ \\
\hline \multicolumn{8}{|l|}{ Atividade física } \\
\hline Ativo suficiente no lazer & 11,0 & 15,7 & 17,4 & 1,42 & $0,95-2,11$ & 1,57 & $1,08-2,27$ \\
\hline \multicolumn{8}{|c|}{ Diagnóstico médico de doenças } \\
\hline Hipertensão arterialc & 28,3 & 14,5 & 16,1 & 0,77 & $0,60-1,00$ & 0,73 & $0,56-0,95$ \\
\hline Diabetes ${ }^{c}$ & 8,2 & 3,1 & 3,7 & 0,61 & $0,35-1,07$ & 0,63 & $0,37-1,09$ \\
\hline Dislipidemiac & 19,6 & 10,7 & 15,9 & 0,85 & $0,63-1,14$ & 1,01 & $0,77-1,33$ \\
\hline \multicolumn{8}{|l|}{ Exames preventivos } \\
\hline Papanicolaou & 94,9 & 96,0 & 96,2 & 1,02 & $0,96-1,08$ & 1,02 & $0,96-1,08$ \\
\hline Mamografia $^{f}$ & 90,3 & 92,4 & 94,5 & 1,02 & $0,94-1,11$ & 1,04 & $0,95-1,14$ \\
\hline \multicolumn{8}{|c|}{ 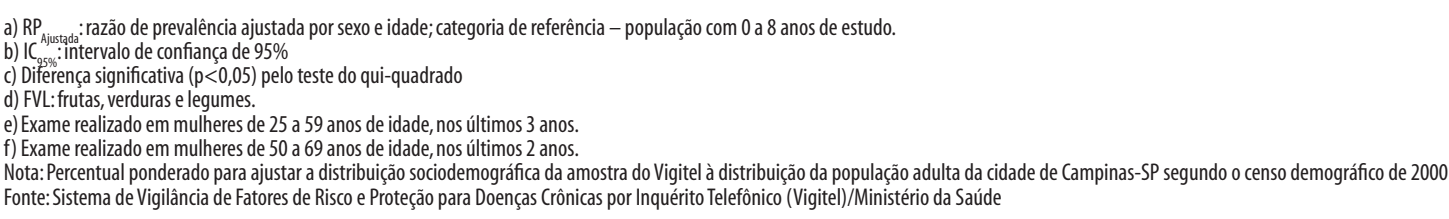 } \\
\hline
\end{tabular}

O tabagismo, a inatividade física, a alimentação inadequada e o uso prejudicial de álcool são os quatro fatores de risco eleitos pela Organização Mundial da Saúde (OMS) para o enfrentamento das doenças crônicas não transmissíveis. ${ }^{1}$ No Brasil, o 'Plano de Ações Estratégicas para o Enfrentamento das Doenças Crônicas Não Transmissíveis 2011-2022' apresenta os quatro principais grupos de doenças - circulatórias, câncer, respiratórias crônicas e diabetes - e seus fatores de risco modificáveis, estabelecendo três eixos de atuação, quais sejam: (i) vigilância, informação, avaliação e monitoramento; (ii) promoção da saúde; e (iii) cuidado integral de doenças crônicas não transmissíveis. Esse Plano, que define metas para a redução das DCNT e de determinantes mediante a valorização de ações de abrangência populacional, apresenta evidências de elevado custo-efetividade. ${ }^{9}$

Nas análises realizadas segundo sexo, observaram-se diferenças para a maioria dos fatores de risco com maiores prevalências entre os homens, exceto para a presença de dislipidemias. Em relação aos fatores de proteção, somente a prática de atividade 
física suficiente no período de lazer foi mais elevada nos homens de Campinas-SP, conforme verificado em outros estudos. ${ }^{4,5,16,17}$

Na população adulta masculina, observou-se maior prevalência de fumantes, não obstante uma redução significativa do hábito de fumar (cessação), acompanhando a tendência verificada em estudo que considerou o período de 2006 a 2009 para a população adulta de capitais brasileiras. ${ }^{18}$ Em relação às faixas etárias, verificou-se prevalência significativamente maior de cessação entre os adultos de 40 ou mais anos, em parte resultante da implementação de políticas regulatórias do Programa de Controle do Tabagismo. ${ }^{8,18}$ A maior prevalência do uso de tabaco entre os menos escolarizados pode ser parcialmente explicada pelo menor acesso a informações sobre seus efeitos. Tais diferenças também foram descritas em outros estudos., ${ }^{4,17,18}$ Para a população adulta brasileira, foi observada maior concentração de fumantes entre indivíduos de menor escolaridade, ${ }^{17,19}$ principalmente homens mais jovens. ${ }^{19}$

0 uso abusivo de álcool revelou prevalência mais elevada entre os homens, conforme observado em outros estudos para a população adulta brasileira. ${ }^{4,16,17}$ Segundo outro inquérito domiciliar também realizado em Campinas-SP, em 2003, com indivíduos de 14 ou mais anos de idade, as prevalências de abuso/dependência de álcool estimadas pelo Alcohol Use Disorder Identification Test (AUDIT) foram maiores nos homens e os padrões de consumo e de dependência diferenciaram-se segundo grupos etários, com menor prevalência entre idosos em relação aos mais jovens (14 a 39 anos), ${ }^{20}$ conforme verificado no presente estudo. Em relação à escolaridade, não foram observadas diferenças significativas quanto ao uso abusivo, 0 que, para Malta e colaboradores, ${ }^{4}$ poderia indicar que o álcool é socialmente aceito e seu uso culturalmente estimulado em todos os níveis de escolaridade. Isto, a despeito das consequências negativas para a saúde e a qualidade de vida geral, aumentando a frequência de enfermidades como hipertensão arterial, diabetes mellitus, cirrose hepática, acidente vascular cerebral, alguns tipos de câncer e transtornos mentais, entre outros, ${ }^{21}$ responsáveis por limitações funcionais, ademais de o consumo abusivo de álcool estar associado a elevados índices de violência e acidentes. ${ }^{16,21}$

$\mathrm{Na}$ avaliação do estado nutricional da população adulta de Campinas-SP, os dados mostraram maior prevalência de sobrepeso entre os homens, como foi observado para a população de 20 a 59 anos de idade em Lages-SC. ${ }^{5}$ Nos adultos com 40 a 59 anos e também nos idosos, as prevalências de sobrepeso e de obesidade foram semelhantes e significativamente maiores em relação aos mais jovens. Em relação à escolaridade, após ajuste por sexo e idade, não houve diferença significativa entre as prevalências de sobrepeso e obesidade segundo os subgrupos avaliados. Estudo recente identificou redução importante de sobrepeso e obesidade na medida do aumento da escolaridade entre as mulheres. ${ }^{4}$ Considerados problemas de saúde mundial, o excesso de peso e a obesidade têm aumentado particularmente nas áreas urbanas de países em desenvolvimento, ${ }^{3}$ elevando o risco de doenças cardiovasculares (doenças cardíacas e acidente vascular cerebral), diabetes, lesões músculo-esqueléticas (especialmente osteoartrite), câncer de endométrio, de mama e de cólon. 0 excesso de peso e a obesidade estão relacionados a mais mortes no mundo do que 0 baixo peso e a desnutrição. ${ }^{15}$

Entre os determinantes do excesso de peso e da obesidade, destacam-se as dietas pouco saudáveis e a inatividade física. As mulheres, assim como os mais idosos e as pessoas com maior escolaridade, são mais cuidadosas com a dieta, ${ }^{4}$ como pode ser verificado pela maior prevalência de consumo regular e recomendado de FVL, também observada em outros estudos, ${ }^{4,5,22}$ e do menor consumo de refrigerantes (ou sucos artificiais) e de carnes com excesso de gordura. ${ }^{5,16}$

Em Campinas-SP, os idosos apresentaram elevadas prevalências de sobrepeso e obesidade, apesar da maior frequência de ingestão diária de frutas e hortaliças e menor ingestão de carnes com excesso de gordura e refrigerantes ou sucos artificiais, possivel reflexo das orientações de profissionais de saúde diante da maior presença de morbidades nesse subgrupo etário. ${ }^{16}$ Neste estudo, entre os adultos jovens, observou-se um elevado consumo de refrigerantes ou sucos artificiais e de carne com excesso de gordura, hábitos semelhantes àqueles verificados para as pessoas de 40 a 59 anos de idade. Os hábitos pouco saudáveis em relação à alimentação são precoces e estão cada vez mais presentes na dieta da população. A prevalência de sobrepeso e obesidade estatisticamente semelhante em relação aos níveis de escolaridade considerados, independentemente do sexo e da idade, sugere a ampliação do consumo de comidas rápidas (fast food) e industrializadas em detrimento de uma 
melhor qualidade da dieta, mesmo entre aqueles com maior acesso à informação. 0 consumo de frutas e hortaliças pelos adultos de Campinas-SP, assim como no Brasil, é muito baixo e a frequência desse consumo não se tem alterado nos últimos anos. ${ }^{23}$

Evidências consistentes demonstram a importância da atividade física para a prevenção de inúmeras doenças na idade adulta. ${ }^{24} \mathrm{~A}$ prática de atividade física foi maior entre os homens e nas pessoas com maior escolaridade; e semelhante em relação às faixas etárias, o que pode ser explicado pela categorização da variável, já que a menor frequência de atividade nas idades mais avançadas é descrita na literatura ${ }^{16}$ A prática de atividade física no período de lazer pelos adultos associa-se, diretamente, ao nível socioeconômico. Nesse sentido, ações como o estímulo da prática em idades precoces podem representar um importante avanço no controle da epidemia de sedentarismo observada em várias partes do mundo e também no Brasil. ${ }^{5,24} \mathrm{O}$ sedentarismo, associado à má qualidade da dieta, manifesta-se em efeitos danosos sobre a saúde das populações. Considerando-se os níveis estáveis e baixos da prática de atividade física observados no país nos últimos anos, ${ }^{25}$ um pequeno aumento do nível de atividade física representaria uma intervenção importante para a melhoria da saúde coletiva, dado seu reconhecido papel na prevenção primária de hipertensão arterial, diabetes tipo 2, doença coronariana, acidente vascular cerebral, osteoporose, alguns tipos de câncer, obesidade e depressão. ${ }^{24}$

Em relação ao diagnóstico médico de doenças, para a população adulta de Campinas-SP, foi observada uma prevalência de dislipidemia de $29 \%$, maior entre as mulheres, independentemente da idade. Observou-se a elevação das prevalências de hipertensão, diabetes e dislipidemias com 0 aumento da idade; e prevalência significativamente menor de hipertensão entre os indivíduos com maior escolaridade, em relação aos menos escolarizados. A elevada concentração de lipídios ou lipoproteínas no sangue é considerada um dos principais fatores de risco para doenças coronarianas, juntamente com a hipertensão, diabetes, sedentarismo, obesidade e tabagismo ${ }^{26}$ A maior prevalência de algumas doenças crônicas nas mulheres e 0 aumento de DCNT - a exemplo da hipertensão e do diabetes ${ }^{27}$ com a idade refletem a maior oportunidade de diagnóstico médico, além do acúmulo de fatores de risco nos mais idosos. A maior procura por serviços de saúde pelas mulheres e idosos, assim como o maior acesso a informações sobre saúde, prática de atividade física e hábitos alimentares mais saudáveis pelas pessoas com melhor nível socioeconômico, podem explicar, parcialmente, tais achados. Ressalta-se que as diferenças entre as prevalências de doenças dos estratos socioeconômicos tendem a ser subestimadas, devido ao menor acesso a serviços médicos e diagnósticos por parte dos segmentos de menor escolaridade. ${ }^{27}$

As prevalências de realização de exames de Papanicolau em mulheres de 25 a 59 anos nos últimos 3 anos, assim como a de mamografia para aquelas com idade entre 50 e 69 anos nos últimos 2 anos, foram elevadas e não apresentaram diferenças segundo a escolaridade. Tal achado revela equidade de acesso a esses exames preventivos no município. Estudo comparativo das estimativas da cobertura de Papanicolau em mulheres de 20 ou mais anos de idade, e de mamografia naquelas acima de 40 anos, a partir de dados de dois inquéritos domiciliares realizados em Campinas-SP (ISA-SP 2001/2002 e ISACamp 2008/2009), verificou aumento estatisticamente significativo das coberturas de ambos os exames no período, independentemente da escolaridade e da idade, ${ }^{28}$ ou seja, uma elevação na probabilidade de diagnóstico precoce do câncer de colo uterino e do câncer de mama para todas as mulheres residentes no município. Na capital do estado de São Paulo, a comparação das prevalências desses mesmos exames preventivos, a partir de dados do ISA-Capital e do Vigitel, encontrou elevadas coberturas de realização de Papanicolau sem diferenças segundo os níveis de escolaridade, enquanto para a realização de mamografia no último ano, observaram-se diferenças entre as mulheres com maior e menor escolaridade, independentemente da modalidade do inquérito (telefônico ou domiciliar). ${ }^{29}$

A restrição da amostra aos indivíduos cobertos por pelo menos uma linha telefônica residencial, o uso de informações autorreferidas e o desenho transversal, que não permite avaliação de causa-efeito, podem ser considerados como limitações do presente estudo. Fatores de ponderação usados pelo Vigitel corrigem parcialmente as diferenças entre as populações com e sem telefone, permitindo que as estimativas sejam extrapoladas à totalidade dos habitantes do município. ${ }^{12,13,16,29}$

Deve-se destacar que 0 aumento da prevalência das doenças crônicas se reflete, fortemente, na elevação da 
procura por serviços e na restrição de atividades pelas pessoas acometidas, com impacto social importante ${ }^{27}$ devido à ampliação do período de tempo no qual os indivíduos permanecem sob essa condição durante seu ciclo de vida. Perspectivas de aumento do número de consultas, exames e cirurgias, outrora delineadas, tornam-se rapidamente concretas com elevado ônus para o Sistema Único de Saúde (SUS), demandas diversas e específicas nos municípios brasileiros, evidenciando a necessidade de organização, qualificação e ampliação do atendimento.

Os resultados desse estudo apontaram diferenças significativas na prevalência de fatores de risco e proteção para DCNT segundo sexo, faixas etárias e escolaridade nos adultos de Campinas-SP, com tendência de comportamentos mais saudáveis entre as mulheres, os idosos e aqueles com maior escolaridade, conforme vem sendo observado em outros estudos sobre dados nacionais. ${ }^{4,16}$

A distribuição dos fatores de risco e proteção à saúde é socialmente determinada. ${ }^{7}$ No Brasil, o SUS, sem comprometer seu caráter universal, tem buscado a promoção da equidade nos serviços de saúde. Entre os princípios organizativos do Sistema, a descentralização territorial é entendida como uma redistribuição das responsabilidades quanto às ações e serviços de saúde entre os vários níveis de governo. ${ }^{30}$ Tanto os gestores quanto os profissionais dedicados ao SUS assumem o importante papel de estimular mudanças nos comportamentos relacionados à saúde das pessoas que procuram pelos serviços no locus onde estão inseridos, promovendo mudanças em uma sociedade identificada sob evidentes desigualdades sociais.

\section{Referências}

1. World Health Organization. 2008-2013 Action plan for the global strategy for the prevention and control of non communicable disease [Internet]. Geneva: World Health Organization; 2008 [cited 2013 Sep 12]. Available from: http://whqlibdoc.who.int/ publications/2009/9789241597418_eng.pdf

2. Schmidt MI, Duncan BB, Azevedo e Silva G, Menezes AM, Monteiro CA, Barreto SM, et al. Chronic non communicable diseases in Brazil: burden and current challenges. Lancet. 2011 Jun;377(9781):1949-61.

3. Malta DC, Silva JB. Policies to promote physical activity in Brazil. Lancet. 2012 Jul;380(9838):195-6.
0 'Plano de Ações Estratégicas para o Enfrentamento das Doenças Crônicas não Transmissíveis no Brasil 2011-2022' propõe ações de abrangência populacional intersetoriais, que extrapolam o setor Saúde.

As ações relacionadas à promoção da saúde $\mathrm{e}$ prevenção de agravos devem estar inseridas nas prioridades do setor Saúde, em todos seus níveis de gestão. ${ }^{9}$

As desigualdades sociodemográficas observadas expressaram-se nos fatores de risco e proteção analisados e diferenciaram-se, com maior ou menor magnitude, entre os segmentos considerados pelas comparações realizadas. Resultados obtidos a partir de dados de inquéritos de saúde de base populacional, como os inquéritos telefônicos, possibilitam, de forma rápida, a identificação de singularidades capazes de contribuir para o estabelecimento de prioridades, alocação de recursos e delineamento de programas voltados à redução das desigualdades sociais em saúde, pautados nas necessidades de cada população.

\section{Contribuição dos autores}

Francisco PMSB contribuiu com a proposta do artigo, revisão de literatura, análise dos dados e redação do manuscrito.

Segri NJ colaborou na análise dos dados e revisão do manuscrito.

Barros MBA e Malta DC orientaram a proposta do artigo e colaboraram na revisão crítica do manuscrito.

Todos os autores aprovaram a versão final do manuscrito e assumem responsabilidade por todos os aspectos do trabalho, incluindo a garantia de sua precisão e integridade.

4. Malta DC, Moura EC, Morais Neto OL. Gender and schooling inequalities in risk and protective factors for chronic diseases among Brazilian adults, through telephone survey. Rev Bras Epidemiol. 2011 Sep;14 Suppl 1:125-35.

5. Longo GZ, Neves J, Castro TG, Pedroso MR, Matos IB. Prevalência e distribuição dos fatores de risco e proteção para doenças crônicas não transmissíveis entre adultos da cidade de Lages (SC), sul do Brasil, 2007. Rev Bras Epidemiol. 2011 dez;14(4):698-708.

6. Beaglehole R, Bonita R, Horton R, Adans C, Alleyne G, Asaria P, et al. Priority actions for the 
non-communicable disease crisis. Lancet. 2011

Apr;377(9775):1438-47.

7. Organização Mundial da Saúde. Diminuindo diferenças: a prática das políticas sobre determinantes sociais da saúde: documento de discussão. In: Conferência Mundial sobre Determinantes Sociais. 2011 out 19-21; Rio de Janeiro. Genebra: Organização Mundial da Saúde; 2011.

8. Wünsch Filho V, Mirra AP, Mendoza López RV, Antunes L. Tabagismo e câncer no Brasil: evidências e perspectivas. Rev Bras Epidemiol. 2010 jun;13(2):175-87.

9. Ministério da Saúde (BR). Secretaria de Vigilância em Saúde. Departamento de Análise de Situação de Saúde. Coordenação Geral de Doenças e Agravos Não Transmissíveis. Plano de ações estratégicas para o enfrentamento das doenças crônicas não transmissíveis (DCNT) no Brasil 2011-2022 [Internet]. Brasília: Ministério da Saúde; 2011 [citado 2013 jul 5]. Disponível em: http://portal. saude.gov.br/portal/arquivos/pdf/cartilha_plano.pdf

10. Olsen J, Bertollini R, Victora C, Saracci R. Global response to non-communicable diseases - the role of epidemiologists. Int J Epidemiol. 2012 Oct;41(5):1219-20.

11. Malta DC, Leal MC, Costa MFL, Moraes Neto OL. Inquéritos nacionais de saúde: experiência acumulada e proposta para o inquérito de saúde brasileiro. Rev Bras Epidemiol. 2008 mai;11(supl 1):159-67.

12. Ministério da Saúde (BR). Secretaria de Vigilância em Saúde. Secretaria de Gestão Estratégia e Participativa. Vigitel Brasil, 2008: vigilância de fatores de risco e proteção para doenças crônicas por inquérito telefônico. Brasília: Ministério da Saúde; 2009.

13. Francisco PMSB, Barros MBA, Segri NJ, Alves MCGP. Comparação de estimativas de inquéritos de base populacional. Rev Saude Publica. 2013 fev;47(1):60-8.

14. Instituto Brasileiro de Geografia e Estatística. Cidades@: São Paulo, Campinas [Internet]. 2013 [citado 2013 mar 3]. Disponível em: http://www.ibge.gov.br/cidadesat/xtras/perfil. php?codmun $=350950 \&$ search $=$ sao-paulo $\mid$ campinas

15. World Health Organization. Obesity and overweight. Fact sheet $\mathrm{n}^{\circ}$ 311. Geneva: World Health Organization; 2011.
16. Iser BPM, Claro RM, Moura EC, Malta DC, Moraes Neto OL. Fatores de risco e proteção para doenças crônicas não transmissíveis obtidos por inquérito telefônico - VIGITEL Brasil - 2009. Rev Bras Epidemiol. 2011 set;14 supl 1:90-102.

17. Moura EC, Malta DC, Moraes Neto OL, Monteiro CA. Prevalence and social distribution of risk factors for chronic non communicable diseases in Brazil. Rev Panam Salud Publica. 2009 jul;26(1):17-22.

18. Azevedo e Silva G, Valente JG, Malta DC. Tendências do tabagismo na população adulta das capitais brasileiras: uma análise dos dados de inquéritos telefônicos de 2006 a 2009. Rev Bras Epidemiol. 2011 set;14 supl 1:103-14.

19. Azevedo e Silva G, Valente JG, Almeida LM, Moura EC, Malta DC. Tabagismo e escolaridade no Brasil, 2006. Rev Saude Publica. 2009 nov;43 supl 2:48-56.

20. Barros MBA, Botega NJ, Dalgalarrondo P, MarínLeón L, Oliveira HB. Prevalence of alcohol abuse and associated factors in a population-based study. Rev Saude Publica. 2007 Aug;41(4):502-9.

21. World Health Organization. Global status report on alcohol and health [Internet]. Geneva: World Health Organization; 2011 [cited 2013 Jul 5] . Available from: http://www.who.int/substance_abuse/publications/ global_alcohol_report/msbgsruprofiles.pdf

22. Serdula MK, Gillespie C, Kettel-Khan L, Farris R, Seymour J, Denny C. Trends in fruit and vegetable consumption among adults in the United States: Behavioral Risk Fator Surveillance System, 19942000. Am J Public Health. 2004 Jun;94(6):1014-8.

23. Souza AM, Bezerra IN, Cunha DB, Sichieri R. Avaliação dos marcadores de consumo alimentar do VIGITEL (2007- 2009). Rev Bras Epidemiol. 2011 set;14 supl 1:44-52.

24. Florindo AA. Atividade física e doenças crônicas. In: Florindo AA, Hallal PC, organizadores. Epidemiologia da atividade física. São Paulo: Atheneu; 2011. p. 159-82.

25. Hallal PC, Knuth AG, Reis RS, Rombaldi AJ, Malta DC, Iser BPM, et al. Tendências temporais de atividade física no Brasil (2006-2009). Rev Bras Epidemiol. 2011 set;14 supl 1:53-60.

26. Sposito AC, Caramelli B, Fonseca FAH, Bertolani MC. IV Diretriz brasileira sobre dislipidemias e prevenção da aterosclerose da Sociedade Brasileira de Cardiologia. Arq Bras Cardiol. 2007 abr;88 supl 1:1-18. 
27. Barros MBA, Francisco PMSB, Zanchetta LM, César CLG. Tendências das desigualdades sociais e demográficas na prevalência de doenças crônicas no Brasil, PNAD: 2003- 2008. Cienc Saude Coletiva. 2011 set;16(9):3755-68.

28. Francisco PMSB, Barros MBA, Segri NJ, Alves MCGP, César CLG, Carandina L, et al. Comparação das estimativas de prevalência de indicadores de saúde no Município de Campinas, São Paulo, Brasil, nos anos de 2001/2002 (ISA-SP) e 2008/2009 (ISA-Camp). Cad Saude Publica. 2013 jun;29(6):1149-60.

29, Segri NJ, Francisco PMSB, Alves MCGP, Barros MBA, Cesar CLG, Goldbaum M, et al. Práticas preventivas de detecção de câncer em mulheres: comparação das estimativas dos inquéritos de saúde (ISA-Capital) e vigilância de fatores de risco e proteção para doenças crônicas por inquérito telefônico (VIGITEL-São Paulo). Rev Bras Epidemiol. 2011 set;14 supl 1:31-43.
30. Brasil. Lei n. 8080, de 19 de setembro de 1990. Dispõe sobre as condições para a promoção, proteção e recuperação da saúde, a organização e o funcionamento dos serviços correspondentes e dá outras providências. Diário Oficial da República Federativa do Brasil, Brasília (DF), 1990 set 20; Seção 1:1.

Recebido em 31/07/2014

Aprovado em 04/12/2014 